\title{
Rendimiento de grupo electrógeno con motor de combustión interna a biogas
}

\section{Electric generation performance with internal combustion engine to biogas}

\author{
Miguel Barrena-Gurbillón ${ }^{1 *}$, Evans Tejedo-Soberón ${ }^{1}$, Edwin Yopán-Chocaca ${ }^{1}$, Meregildo Silva-Ramírez $^{1}$
}

\section{RESUMEN}

Se determinó el rendimiento de grupo electrógeno de $1 \mathrm{~kW}$ con motor de combustión interna de 2,4 hp a biogas en vez de gasolina, en la Estación Experimental Pomacochas del INDES-CES, con biogas producido en biodigestor tubular de $12 \mathrm{~m}^{3}$, con $9 \mathrm{~m}^{3}$ de mezcla estiércol de vacuno:agua (1:5) y $3 \mathrm{~m}^{3}$ para almacenamiento de biogas, con balón adicional de $2 \mathrm{~m}^{3}$. Para mantener la producción de biogas se alimentó diariamente el biodigestor con $200 \mathrm{~L}$ de mezcla estiércol:agua (1:5). La presión de biogas fue de $4 \mathrm{~cm}$ de agua. Para hacer funcionar el motor con biogas, se hizo un dispositivo en forma de T, con accesorios de PVC de $1 / 2$ " y llave de compuerta de bronce de $1 / 2$ " para regular el paso de biogas. Para el ingreso de la mezcla biogas-aire al carburador se empleó accesorios de PVC de 1,5". El grupo electrógeno a biogas produjo $244 \mathrm{~W}(24,4 \%$ de la potencia generada por el mismo motor a gasolina), para un foco de filamento de $100 \mathrm{~W}$ y 8 focos ahorradores de $18 \mathrm{~W}$ cada uno. El arranque del motor se hizo con gasolina y enseguida se pasó a operación con biogas.

Palabras clave: adaptación de motor a biogas, rendimiento de motor a biogas.

\section{ABSTRACT}

The performance of a $1 \mathrm{~kW}$ generator set with an internal combustion engine of 2,4 hp to biogas instead of gasoline was determined at the Experiment Station Pomacochas of INDES-CES, with biogas produced in a $12 \mathrm{~m}^{3}$ tubular biodigester with $9 \mathrm{~m}^{3}$ of manure mix:water $(1: 5)$ and $3 \mathrm{~m}^{3}$ for biogas storage, with additional balloon of $2 \mathrm{~m}^{3}$. To maintain biogas production, the biodigester was fed daily with $200 \mathrm{~L}$ of manure:water mixture (1:5). The pressure of biogas was $4 \mathrm{~cm}$ of water. To operate the engine with biogas, a T-shaped device was made with $1 / 2$ " PVC fittings and $1 / 2$ " brass gate wrench to regulate the biogas flow. For the admission of the biogas-air mixture to the carburetor, 1,5" PVC fittings were used. The biogas generating set produced $244 \mathrm{~W}(24,4 \%$ of the power generated by the same gasoline engine), for a filament bulb of $100 \mathrm{~W}$ and 8 bulbs of $18 \mathrm{~W}$ each. The engine was started with gasoline and was then switched to biogas operation.

Key words: motor adaptation to biogas, motor performance to biogas.

\footnotetext{
Universidad Nacional Toribio Rodríguez de Mendoza de Amazonas (UNTRM-A), Instituto de Investigación para el Desarrollo Sustentable de Ceja de Selva, Calle Higos Urco N ${ }^{\circ} 342-350-356$, Calle Universitaria Nº304, Chachapoyas, Perú

Autor de correspondencia. E-mail: mbarrena@indes-ces.edu.pe
} 


\section{INTRODUCCIÓN}

El biogás contiene entre 40 a 70\%(v/v) de metano y 0,1 $\%(\mathrm{v} / \mathrm{v})$ de $\mathrm{H}_{2} \mathrm{~S}$ (Cepero et al., 2013). El biogás es un combustible ecológico producido por fermentación de la mezcla estiércol:agua en biodigestor anaeróbico. Es adecuado para cocinas, lámparas de camiseta, calefacción y motores de combustión interna en reemplazo de la gasolina y parcialmente del petróleo diesel. El $\mathrm{H}_{2} \mathrm{~S}$ del biogás se elimina haciéndolo pasar por un cartucho relleno con clavos de fierro (Barrena et al., 2013).

En la Universidad EARTH de Costa Rica, para adaptar un motor a gasolina de cuatro tiempos, de $5 \mathrm{hp}$, elaboraron una pieza para introducir la mezcla biogás-aire al cilindro del motor. La parte principal es la T de PVC, donde se mezcla el biogás con aire (Figura 1). Sustituyeron el $100 \%$ de la gasolina por biogás; por cada hora de uso del motor con biogás se ahorra 2213,59 mL de gasolina. El consumo de biogás fue de $0,55 \mathrm{~m}^{3} / \mathrm{kWh}$. Para este motor, $1 \mathrm{~m}^{3}$ de biogás equivale a 4,025 litros de gasolina (Carmona et al., 2007). No se indica la presión del biogás.

En el Instituto de Motores de Combustión Interna (IMCI) de la Universidad Nacional de Ingeniería, Lima (Perú); se hizo funcionar a biogás un motor gasolinero de 8 hp. Para el ingreso de biogás no se modificó el sistema de alimentación de gasolina ni del regulador de velocidad del motor. La potencia máxima del motor con biogás fue de $4,25 \mathrm{~kW}$, que es el 71,2\% de la potencia nominal del motor $(5,97 \mathrm{~kW})$ trabajando con gasolina. Las pruebas con biogás dieron un consumo de 0,65-0,70 $\mathrm{m}^{3} /(\mathrm{kW} . \mathrm{h})$ a plena carga (IMCI, 2012).

Un motor de combustión interna de $20 \mathrm{~kW}$ a biogás generó $18 \mathrm{~kW}$ de energía eléctrica. Su operación se mantuvo por períodos de 1 hora con variaciones de presión del biogás en el biodigestor entre 45 a $155 \mathrm{~cm}$ de agua, consumiendo en promedio $4,7 \mathrm{~m}^{3}$ de biogás a un ritmo de 78,3 L/minuto y un consumo específico de $0,261 \mathrm{~m}^{3} / \mathrm{kWh}$. Motores de ciclo Otto a biogás tuvieron un consumo específico de 0,6 a $0,8 \mathrm{~m}^{3} / \mathrm{kWh}$. La variación fue por cambios permanentes de presión de suministro de biogás (Hernández, 1996).

El petróleo y la gasolina son combustibles ampliamen- te utilizados a nivel mundial para la generación de energía eléctrica. En reemplazo de ellos, en la presente investigación se adaptó el motor a gasolina de un grupo electrógeno para que funcione a biogás, lo cual fue factible debido a que este tipo de motor tiene bujía para generar la chispa que inicia la combustión en el cilindro.

\section{MATERIALYMÉTODOS}

\section{Sistema de producción de biogás}

La Estación Experimental Pomacochas del Instituto de Investigación para el Desarrollo Sustentable de Ceja de Selva (INDES-CES), de la Universidad Nacional Toribio Rodríguez de Mendoza de Amazonas (UNTRM), en Pomacochas, Bongará, Región Amazonas (Perú), tiene en operación un sistema de producción de biogás, instalado en un ambiente cerrado y techado para protegerlo del clima frío (2200 $\mathrm{msnm}$, temperatura ambiente promedio de $15^{\circ} \mathrm{C}$ ).

\section{Grupo electrógeno}

Se empleó un grupo electrógeno con motor de 2,4 hp, a gasolina, con generador de $1000 \mathrm{~W}$.

\section{Potencia del grupo electrógeno a gasolina}

Un cilindro de jeringa hipodérmica de $60 \mathrm{ml}$ con $50 \mathrm{ml}$ de gasolina, se conectó al carburador para medir el tiempo de funcionamiento del motor con ese volumen de gasolina, con una carga de $1000 \mathrm{~W}$ (10 focos de 100 W cada uno).

\section{Potencia del grupo electrógeno a biogás}

Las pruebas se realizaron regulando la entrada de biogás con el dispositivo de mezcla diseñado, hasta encontrar la proporción adecuada que mantenga el motor en funcionamiento estable. En ese momento se iniciaron las pruebas con una carga mínima de $100 \mathrm{~W}$ y una máxima de $1000 \mathrm{~W}$, para esto se utilizó un foco de $100 \mathrm{~W}$ y luego los 9 focos de $100 \mathrm{~W}$ cada uno, del tablero de prueba, que se fueron encendiendo uno tras otro.

\section{Determinación del rendimiento del motor}

Se determinó el consumo de gasolina y de biogás del motor del grupo electrógeno con diferentes cargas. Se midió el tiempo de funcionamiento con una presión de 
biogás en el sistema de producción, expresada en cm de agua. Con estos resultados se calculó el rendimiento del motor con la fórmula:

$$
\text { Rendimiento }=\frac{\text { Litros de combustible }}{\text { Watt } \times \text { minuto }}
$$

\section{RESULTADOS}

\section{Biogás disponible}

El biodigestor de $12 \mathrm{~m}^{3}$ de la EE Pomacochas, tiene disponible $5 \mathrm{~m}^{3}$ de biogás. Se alimentó diariamente con 2001 de mezcla estiércol:agua (1:5) para mantener la producción diaria promedio de $2 \mathrm{~m}^{3}$ de biogás a una presión de $4 \mathrm{~cm}$ de agua.

\section{Potencia del grupo electrógeno a gasolina}

El grupo electrógeno de $1000 \mathrm{~W}$ con $50 \mathrm{ml}$ de gasolina abastecida del cilindro de una jeringa hipodérmica conectado al carburador, funcionó durante 4 minutos con una carga constante de $1000 \mathrm{~W}$ (10 focos de $100 \mathrm{~W}$ cada uno).

\section{Potencia del grupo electrógeno a biogás}

Se realizaron tres pruebas para lograr la adaptación final del grupo electrógeno de $1000 \mathrm{~W}$ a biogás. El encendido del motor siempre se hizo con gasolina y se continuó su funcionamiento con biogás. En la primera prueba se inyectó directamente el biogás al filtro de aire del motor con una manguera de $1 / 4$ ", regulado por una llave de bronce (Figura 4). Se aplicó una carga de $1000 \mathrm{~W}$ (10 focos de $100 \mathrm{~W}$ cada uno), pero el motor no llegaba a un funcionamiento estable a pesar de estar acelerado manualmente al máximo. Al alimentarse el biogás directamente al múltiple de admisión, el ajuste del motor por incremento de la carga se realiza manualmente con la llave de bronce que controla el paso de biogás.

Para la segunda prueba se reemplazó el filtro de aire por un dispositivo en $\mathrm{T}$, hecho con una te de PVC de 1,5 " a la que se conectó una llave de compuerta de bronce de $1 / 2$ " para regular el flujo de biogás abastecido con manguera de $1 / 2$ " y en la otra rama de la te se conectó una llave de paso de 1,5" de plástico para regular el ingreso de aire para la combustión. Se encendió uno por uno los focos de $100 \mathrm{~W}$, llegando hasta tres focos con funcionamiento estable del motor a biogás, comprobándose que sólo puede generar 300 $\mathrm{W}, 30 \%$ de la potencia que genera con gasolina, el combustible para el que está diseñado.

Para la tercera prueba se mejoró el dispositivo de ingreso de biogás y de aire al carburador (Figura 1) en forma de T con accesorios de PVC para mezclar el biogás con el aire en la proporción adecuada para inyectarla a la cámara de combustión del motor. Con la llave de compuerta de bronce se reguló finamente el flujo del biogás. El dispositivo se instaló en el lugar del filtro de aire del carburador. Debido a los resultados previos, se cambió los focos de filamento de $100 \mathrm{~W}$ por focos ahorradores de $18 \mathrm{~W}$ cada uno. El motor tuvo un funcionamiento estable con biogás, haciendo encender siete focos ahorradores del tipo mencionado (126 W en total, representa el $12,6 \%$ de su potencia nominal) (Figura 6); enseguida, se encendieron 8 focos ahorradores de $18 \mathrm{~W}$ y uno de $100 \mathrm{~W}(244 \mathrm{~W}$ en total, representa el $24,4 \%$ de su potencia nominal).

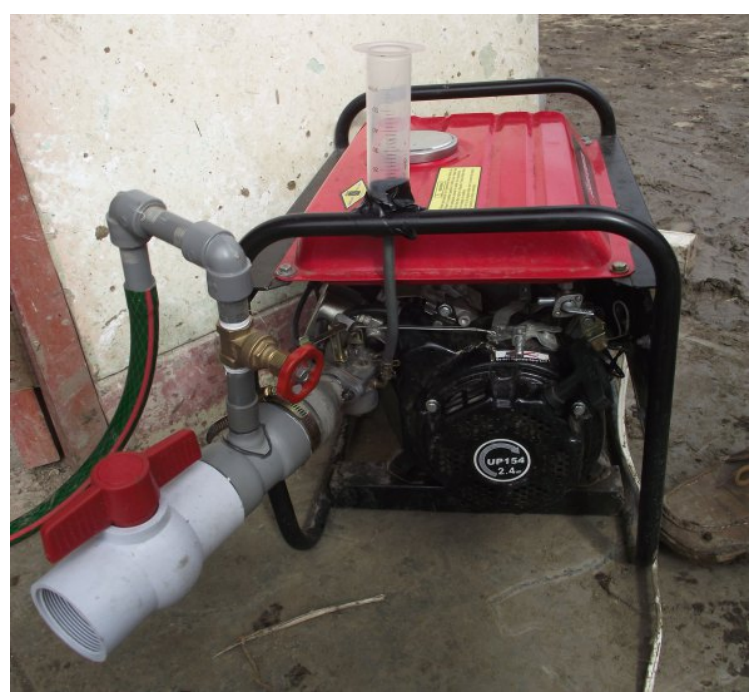

Figura 1. Tercera prueba. Grupo electrógeno de $1 \mathrm{~kW}$, generando $244 \mathrm{~W}$ a biogas.

La secuencia de operación del motor a biogás debe ser la siguiente:

a) Verificar que la presión del biogás esté como mínimo a $4 \mathrm{~cm}$ de agua.

b) Utilizar gasolina para el arranque y durante el calentamiento del motor, de 3 a 5 minutos, según 
las condiciones ambientales.

c) Acelerar el motor antes de cambiar de gasolina a biogás. Es más fácil hacer este cambio con el motor acelerado, que a bajas revoluciones. Una vez hecho el cambio se podrá ajustar a las revoluciones requeridas.

d) Cerrar el paso de gasolina al carburador. Esperar que se termine la gasolina del carburador y, en el momento en que el motor esté por detenerse, se abre lentamente la llave de paso del biogás al motor. Esto evitará que el motor succione demasiado biogás y se detenga por una mezcla (aire y biogás) demasiada rica.

e) Regular el funcionamiento del motor con la apertura de la llave de compuerta para dejar fluir el biogás, de acuerdo a lo que necesite el motor.

\section{Rendimiento del motor con gasolina}

El motor de 2,4 hp del grupo electrógeno de $1000 \mathrm{~W}$, consume $50 \mathrm{ml}$ de gasolina en 4 minutos con lo que genera energía eléctrica para el funcionamiento de 10 focos de $100 \mathrm{~W}$ cada uno, es decir $1000 \mathrm{~W}$.

$$
\text { Rendimiento }=\frac{0.05 \text { Litros de combustible }}{1000 \text { Watt } \times 4 \text { minuto }}
$$

Rendimiento $=1,25 \times 10^{-5} 1$ gasolina $/ \mathrm{W} \times \min =$ $7,5 \times 10^{-4} \mathrm{~m}^{3}$ gasolina $/ \mathrm{kW} \times \mathrm{h}$

\section{Rendimiento del motor con biogás}

Este mismo motor funcionando con biogás consume 20001 en 30 minutos, para producir $244 \mathrm{~W}$ de energía eléctrica ( 8 focos ahorradores de $18 \mathrm{~W}$ y 1 foco de 100 W). La presión inicial del biogás fue de $4 \mathrm{~cm}$ de agua y la presión final fue de $1 \mathrm{~cm}$ de agua.

$$
\text { Rendimiento }=\frac{2000 \text { Litros de combustible }}{244 \text { Watt } \times 30 \text { minuto }}
$$

Rendimiento $=0,2731$ biogás $/ \mathrm{W} \times \min =16,38 \mathrm{~m}^{3}$ de biogás $/ \mathrm{kW} \times \mathrm{h}$ )

El rendimiento del motor con gasolina fue de $16,38 / 7,5 \times 10^{-4}=21840$ veces mayor que con biogás. Para mejorar el rendimiento se debe mantener constante la presión de biogás.

\section{DISCUSIÓN}

Debido a que el biogás contiene aproximadamente $60 \%$ de metano, presenta ciertas desventajas de operación con respecto a la gasolina, que es un combustible con mejores características que el biogás. El motor funcionando con biogás no soporta demasiada carga. La solución sería suministrarle mayor cantidad de biogás, pero esto no es posible debido a que existe un volumen constante de aire-combustible dentro del cilindro del motor, y al introducirle más biogás lo que se hace es alterar en grandes rangos la relación de mezcla, como consecuencia el motor se apaga. La cantidad de biogás necesaria para que el motor trabaje normalmente y a una determinada carga, es solicitada automáticamente por la depresión creada en la succión, ya que el motor dispone de un control de velocidad constante. El motor debe trabajar con carga constante para que no se presenten estos inconvenientes.

En los motores de ciclo Otto, el carburador convencional es reemplazado por un mezclador de gases. Estos motores son encendidos con gasolina y luego siguen funcionando con $100 \%$ de biogas, con una merma de potencia del 20\% al 30\% (Escobedo, 2011). En la presente investigación, con la adaptación realizada, no se modificaron los componentes del motor y podrá utilizar biogás o gasolina.

No se requiere alta presión para introducir al cilindro el biogás mezclado con el aire, ya que la succión del motor (admisión), permite la entrada de la mezcla de gases. Sin embargo, es necesario que la presión del biogás se conserve constante.

Los motores a gasolina son de combustión interna y por tener una baja relación de compresión, no se puede aprovechar todo el potencial calorífico del biogás. Se lograría si al motor se le incrementa su relación de compresión, lo que requiere modificaciones especializadas y también daría problemas cuando por alguna razón falte el biogás, pues el motor modificado sólo funcionaría con biogás y no con gasolina (Escobedo, 2011).

En el motor de 2,4 hp del grupo electrógeno de $1000 \mathrm{~W}$ adaptado a biogás utilizado en las pruebas de la 
presente investigación, la potencia se reduce a $244 \mathrm{~W}$, lo que corresponde al $24,4 \%$ de la potencia de $1000 \mathrm{~W}$ cuando funciona con gasolina. Por lo que la adaptación de motores de combustión interna de cuatro tiempos, de gasolina a biogás, tiene el inconveniente que la potencia útil del motor se reduce. Sin embargo, por ser el biogás un combustible renovable, barato y de fácil producción contribuye a reducir el uso de combustibles derivados del petróleo.

El biogás reduce casi cuatro veces la potencia que genera con gasolina el motor de combustión interna del grupo electrógeno, por ello, es mejor emplear focos ahorradores de $18 \mathrm{~W}$ que iluminan como un foco de filamento de $80 \mathrm{~W}$. De esta manera, se hace doble contribución al cuidado del medio ambiente; en la primera, se combustiona el metano del biogás en el cilindro del motor, con lo que una molécula de $\mathrm{CH}_{4} \mathrm{se}$ convierte en una molécula de $\mathrm{CO}_{2}$, reduciendo en 24 veces su poder como gas de efecto invernadero; en la segunda, los focos ahorradores disipan menos calor al ambiente que los focos de filamento.

Para mejorar el rendimiento del motor a biogás se debe incrementar la presión de biogás. Para mantener estable la operación del motor se debe mantener constante la presión de biogás. Con este grupo electrógeno a biogás, se ahorra $750 \mathrm{~mL}$ de gasolina por hora de funcionamiento, siendo su motor de $87 \mathrm{~cm}^{3}$. Según Carmona et al. (2007), un motor de 5 hp de 144 $\mathrm{cm}^{3}$, por cada hora de funcionamiento a biogás ahorra 2213,59 mL de gasolina. Al establecer la comparación, resulta que la relación de volúmenes de cilindrada es de 144/87 = 1,66; sin embargo, la relación de volumen de gasolina gastado es 2213,59/750 = 2,95. En consecuencia, el gasto de gasolina del motor de $5 \mathrm{hp} \mathrm{es}$ 2,95 veces más, lo que concuerda por ser el doble de potencia que el motor de $2,4 \mathrm{hp}$.

En la presente investigación, la potencia del motor de 2,4 hp se redujo a $24,4 \%$; a diferencia de lo que ocurre con un motor de 2,3 veces más potencia ( $8 \mathrm{hp}$ ), cuya potencia con biogás fue de $71,2 \%$ de su potencia con gasolina (IMCI, 2012).

Un motor de 5 hp a biogás tiene un rendimiento de 0,55 $\mathrm{m}^{3} /(\mathrm{kWh})$ (Carmona et al., 2007); mientras que para un motor de $8 \mathrm{hp}$, su rendimiento es de 0,65 a 0,70 $\mathrm{m}^{3} /(\mathrm{kWh}$ ) (IMCI, 2012). Para ambos casos no hay un reporte de la presión del biogás empleado. Sin embargo, Hernández (1996), reportó que con biogás a una presión entre 45 a $155 \mathrm{~cm}$ de agua, el rendimiento de un motor de $20 \mathrm{~kW}$ generando $18 \mathrm{~kW}$ de energía eléctrica fue de $0,261 \mathrm{~m}^{3} /(\mathrm{kWh})$. Para la presente investigación, se empleó biogás a una presión entre 1 a $4 \mathrm{~cm}$ de agua y el rendimiento del motor de 2,4 hp fue de $16,38 \mathrm{~m}^{3} /(\mathrm{kWh})$, empleando la relación $\mathrm{P} 1 \mathrm{~V} 1=\mathrm{P} 2 \mathrm{~V} 2$ (a temperatura constante): (155 $\mathrm{cm})\left(0,261 \mathrm{~m}^{3} /(\mathrm{kWh})\right)=(4 \mathrm{~cm}) \mathrm{V} 2 ; \mathrm{V} 2=10,11$ $\mathrm{m}^{3} /(\mathrm{kWh})$; se aprecia que el rendimiento es $38 \%$ menor, por lo que se refuerza la conclusión que los motores a biogás funcionarán mejor cuando se alimenten con biogás a mayor presión, la que además debe mantenerse constante.

La aplicación de esta tecnología en zonas rurales es factible puesto que se requiere un mínimo de cuatro vacas para tener el estiércol necesario para alimentar el biodigestor cada día y mantener la producción de biogás constante. Permitirá a los usuarios tener energía eléctrica para iluminación y para el funcionamiento de un televisor o una radio, por lo que la adaptación del motor a gasolina del grupo electrógeno para que pueda funcionar a biogás, contribuirá a mejorar la calidad de vida del poblador rural.

\section{CONCLUSIONES}

Se ha demostrado que es factible la adaptación de un motor a gasolina, al uso de biogás; aunque reduce su potencia en cuatro veces.

Se mantuvo la integridad del motor, no se alteró sus mecanismos, como hubiese sido el caso de la adaptación de un carburador especial para biogás. Se ha empleado un carburador normal para gasolina, y sin modificaciones ha funcionado con biogás.

A mayor presión de biogás, funcionamiento más estable del motor, siempre y cuando se mantenga constante la presión de abastecimiento de biogás. 
El empleo de focos ahorradores permite compensar la disminución de la potencia del grupo electrógeno a biogás.

Es una tecnología factible de ser empleada en zonas rurales para mejorar la calidad de vida de sus usuarios.

\section{RECOMENDACIONES}

Dar arranque al motor con gasolina hasta que caliente, después pasar a alimentación con biogás, abriendo lentamente la válvula de la tubería de biogás y al mismo tiempo cerrar el paso de la gasolina.

Mantener constante la presión del biogás alimentado al motor.

Mantener el motor con la misma carga siempre que sea posible, para evitar regulaciones frecuentes de la válvula de entrada del biogás, lo que interfiere con el funcionamiento del motor y baja su rendimiento.

Difundir la tecnología del biogás, resaltando sus beneficios.

\section{AGRADECIMIENTOS}

Al Proyecto SNIP 352431 "Creación de los Servicios del Centro de Investigación en Climatología y Energías Alternativas" (PROCICEA) del INDES-CES de la Universidad Nacional Toribio Rodríguez de Mendoza, Región Amazonas, por el apoyo para realizar la presente investigación.

\section{REFERENCIAS BIBLIOGRÁFICAS}

Barrena, M. A., L. A. Taramona Ruiz, O. A. Gamarra Torres y M. C. Wong. 2013. Biodigestores tubulares para la producción de biogás. Lima (Perú): GRAFICADAVISA.

Carmona, J. O., R. Botero y C. Hernández. 2007. Utilización de biogás para operar motores a gasolina y diésel. San José (Costa Rica): Universidad EARTH. Recuperado de: w w w. e n g o r m i x . c o m / M A porcicultura/manejo/articulos/utilizacionbiogás-operar-motorest1212/p0.htm.

Cepero, L., V. Savran, D. Blanco, M. R. Díaz Piñón, J. Suárez y A. Palacios. 2013 "Producción de biogás y bioabonos a partir de efluentes de biodigestores". Pastos y Forrajes 35. Recuperado de: http://scielo.sld.cu/scielo.php ?script=sci_arttext\&pid=S0864-039420120 00200009\&Ing=es\&nrm=iso.

Escobedo, J. R. 2011. Biogás como combustible alternativo para una estufa y un motor de un cilindro ciclo Otto. Tesis de Grado. Universidad de San Carlos de Guatemala. Guatemala (Guatemala).

Hernández, L. A. 1996. "Tecnologías para el aprovechamiento del gas producido por la digestión anaeróbica de la materia orgánica”. Agronomía Colombiana 13: 76-90. 